\title{
TRANSVERSE TRIPOLAR STIMULATION FOR SELECTIVE FNS
}

\author{
Kirsten E.I. Deurloo, and Jan Holsheimer \\ Institute for Biomedical Technology \\ Department of Electrical Engineering, Biomedical Engineering Division \\ University of Twente, P.O. Box 217, 7500 AE Enschede, The Netherlands \\ e-mail: k.e.i.deurloo@el.utwente.nl,j.holsheimer@el.utwente.nl
}

\begin{abstract}
Various anode-cathode configurations in a nerve cuff were modeled in order to optimize its spatial selectivity characteristics for FNS. Apart from usual configurations (monopole, bipole, longitudinal tripole, "steering" anode), a transverse tripolar configuration (central cathode) was examined. The model predictions were verified by acute animal experiments. It is concluded that a transverse tripole activates superficial nerve fibers in a more selective way than other configurations do.
\end{abstract}

\section{INTRODUCTION}

Functional Neuromuscular Stimulation (FNS) is a technique to restore motor functions in patients with paralysis caused by spinal cord injuries and other central neurological deficits. The paralyzed muscle can be activated by electrical stimulation of the corresponding peripheral motor nerve fibers. To produce muscle activation resulting in "normal" motor behavior, independent control of motor neuron pools is necessary. Therefore, electrode devices stimulating these neuron pools in a selective way are needed.

Several studies are published in which the spatial selectivity was tested when using various electrode configurations of a nerve cuff containing twelve "dot" electrodes aligned as four longitudinal tripoles [1,2,3]. Best spatial selectivity was achieved when a longitudinal tripole with a "steering" anode was used. The selectivity was maximized when the transverse current of the "steering" anode was a high proportion of the total current. Accordingly, maximum spatial selectivity might be obtained when using a $100 \%$ transverse current.

In modeling spinal cord stimulation, it was shown that a transverse tripolar electrode configuration (central cathode) might successfully be used to selectively stimulate a dorsal column area [4]. It was shown that in this way the recruited area in the dorsal columns was less wide for the same depth than at stimulation with a longitudinal configuration. Because this shape of the recruited area may correspond better to the shape of a nerve fascicle, we analyzed theoretically the capability of transverse tripolar electrode configurations in a nerve cuff for selective FNS. The model predictions were verified by acute experiments on rabbits.

\section{METHODS}

A two-part computer model was used for the simulations [5]. The first part is a three-dimensional volume conductor model representing the nerve and cuff electrode. A monofascicular nerve with a length of $23 \mathrm{~mm}$ and a diameter of $1.9 \mathrm{~mm}$, surrounded by a $50 \mu \mathrm{m}$ layer of perineurium, a $50 \mu \mathrm{m}$ layer of epineurium, and a cylindrical insulating cuff electrode $(2.2 \mathrm{~mm}$ inner diameter, $0.25 \mathrm{~mm}$ thick. $5 \mathrm{~mm}$ long) was modeled, see fig. la. The nerve and cuff were surrounded by saline. Electrode contacts were $1.0 \mathrm{~mm}$ long and $0.5 \mathrm{~mm}$ wide, and were defined as current sources. In this model, the potential distribution resulting from stimulation by any particular contact configuration was calculated. This was done by solving the discretized Laplace equation using a Red-Black Gauss-Seidel iterative method with variable overrelaxation.

The second part of the computer model enables the calculation of the threshold current for excitation of individual myelinated nerve fibers within the fascicle by a McNeal-type fiber model.

Acute experiments were performed on rabbits. An insulating stimulating cuff (i.d. $=1.5 \mathrm{~mm}$ ) with five electrode contacts, equally spaced in a transverse plane. was implanted on the left sciatic nerve. $\mathrm{M}$-waves were recorded by wire electrodes, placed in several calf muscles. In this way, the selectivities obtained by a monopole, a transverse bipole, and a transverse tripole electrode configuration were quantified.

\section{RESULTS}

Figure 1 shows the recruitment contours for three electrode configurations. At three fixed positions in the fascicle in front of the cathode at $0.3,0.5$, and $0.7 \mathrm{~mm}$ distance, the threshold current to excite a $15 \mu \mathrm{m}$ fiber with a rectangular current pulse of $350 \mu \mathrm{sec}$, and the corresponding recruitment contour were calculated. When stimulation is given unipolarly (fig. 1a) or with a longitudinal tripole (not shown), the contours are concave and the current is spreading along the circumference of the nerve bundle (due to the shunting saline layer between nerve and cuff). When a 


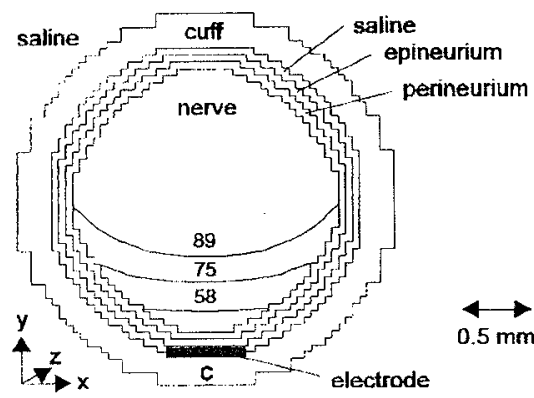

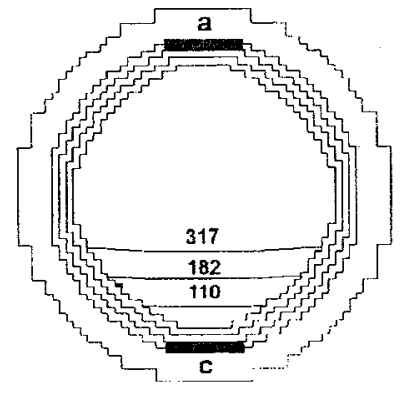

b

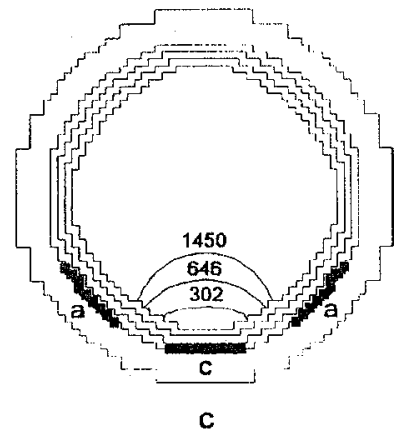

Figure 1: Recruitment lines showing excitation regions in the nerve trunk. The corresponding threshold currents are given (cathodal current in $\mu$ A). $\mathrm{a}=$ single cathode, $\mathrm{b}=$ transverse bipole, $\mathrm{c}=$ transverse tripole
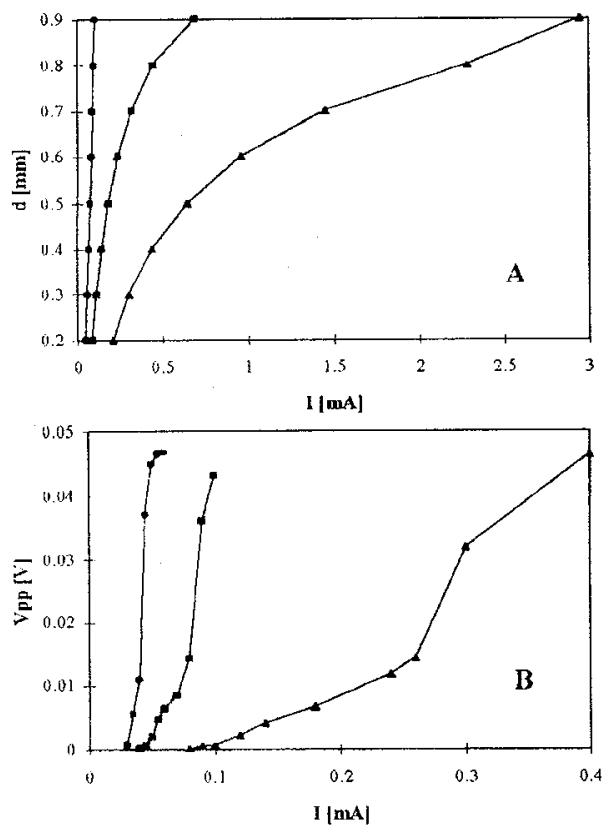

Figure 2: (a) Distance to the cathode of a $15 \mu \mathrm{m}$ fiber as a function of threshold current (simulation). (b) Peak-to-peak amplitude of $M$-wave as a function of stimulation current (experiment). $\bullet=$ monopole, $\mathbf{a}=$ bipole, $\boldsymbol{\Lambda}=$ tripole

longitudinal tripole with a "steering" anode is used, the recruitment contours get less concave, and almost straight when $100 \%$ steering current (= bipole, see fig. $1 \mathrm{~b}$ ) is used. More current is now needed to excite the fibers. A transverse tripole (fig. 1c) results in convex recruitment contours. Threshold currents are highest for this configuration and increase more steeply with fiber-cathode distance. This is also shown in fig. $2 \mathrm{a}$ where this distance is given as a function of the threshold current for the three configurations of fig. 1. When the contact spacing of the transverse tripole is increased, less current is needed, but the contours get wider. Increasing the contact area has similar effects.

Figure $2 b$ shows the peak-to-peak amplitude of the $M$ wave measured in the lateral gastrocnemius of a rabbit as a function of stimulation current, while stimulating with a monopole, a transverse bipole and a transverse tripole (the cathode was at the tibial side of the sciatic nerve). For the monopole, small threshold currents were needed and the recruitment curve was steep. For the bipole, slightly more current was needed and the recruitment curve was less steep. For the tripole, threshold currents were highest and the recruitment curve was least steep. Comparing the data of different muscles and different contacts as the cathode from the same experiment shows that the transverse tripole is more selective than the other configurations (data not shown).

\section{DISCUSSION}

Modeling has shown that a transverse tripolar configuration gives a nerve recruitment area fitting best to the shape of nerve fascicles. It would thus enable sclective activation of a fascicle in the periphery of a nerve trunk. without activating neighboring fascicles.

If the fiber-cathode distance is taken as a measure of the number of recruited nerve fibers, it would also relate to the peak-to-peak amplitude of the $\mathrm{M}$-wave. Indeed, the curves in figures $2 \mathrm{a}$ and $2 \mathrm{~b}$ are similar. The experimental data (fig. 2b) confirm that with the transverse tripole threshold currents are relatively high and the recruitment curve is less steep than for other configurations. Detailed modeling of experimental results is still needed to optimize the transverse tripole configuration.

\section{REFERENCES}

I1) C. Veraart, W.M. Grill, and J.Y. Mortimer, "Selective control of muscle activation with a multipolar nerve cuff electrode." IEEE Trans. Biomed. Eng., vol. 40, pp. 640-653, 1993.

[2] J.D. Sweeney, N.R. Crawtord, and T.A. Brandon. "Neuromuscular stimulation selectivity of multiple-contact nerve cuff electrode arrays," Med. Biol. Eng. Comp., vol. 33, pp. 418-425, 1995.

[3] E.V. Goodall, F. de Breij, and J. Holsheimer, "Position-selective activation of peripheral nerve fibers with a cuff electrode," IEEE Trans. Bioned. Eng. in press.

[4] J.J. Struijk, and J. Holsheimer, "Transverse tripolar spinal cord stimulation Theoretical performance of a dual channel system," Med. Biol. Erg. Comp., in press.

[5] J.J. Struijk, J. Holsheimer, G.G. van der Heide, and H.B.K. Boom, "Recruitment of dorsal column fibers in spinal cord stimulation," IEEE Trans. Biomed. Eng., vol. 39, pp. 903-912, 1992. 